\title{
Genetic module and miRNome trait analyses reflect the distinct biological features of endothelial progenitor cells from different anatomic locations
}

Cheng-Chung Cheng ${ }^{1}$, Hung-Hao Lo ${ }^{2}$, Tse-Shun Huang $^{2}$, Yi-Chieh Cheng ${ }^{2}$, Shi-Ting Chang ${ }^{3}$, Shing-Jyh Chang ${ }^{4}$ and Hsei-Wei Wang $2,3,5,6^{*}$

\begin{abstract}
Background: Endothelial progenitor cells (EPCs) play a fundamental role in post-natal vascular repair, yet EPCs from different anatomic locations possess unique biological properties. The underlying mechanisms are unclear.

Results: EPCs from CB expressed abundant genes involved in cell cycle, hypoxia signalling and blood vessel development, correlating with the phenotypes that CB-EPCs proliferated more rapidly, migrated faster, and formed tubule structure more efficiently. smRNA-seq further deciphered miRNome patterns in EPCs isolated from CB or PB: 54 miRNAs were enriched in CB-EPCs, while another 50 in PB-EPCs. Specifically, CB-EPCs expressed more angiogenic miRNAs such as miR-31, while PB-EPCs possessed more tumor suppressive miRNAs including miR-10a. Knocking down miR-31 levels in CB-EPCs suppressed cell migration and microtubule formation, while overexpressing miR-31 in PB-EPCs helped to recapitulate some of CB-EPC functions.

Conclusions: Our results show the foundation for a more detailed understanding of EPCs from different anatomic sources. Stimulating the expression of angiogenic microRNAs or genes in EPCs of low activity (such as those from patients with cardiovascular diseases) might allow the development of novel therapeutic strategies.
\end{abstract}

\section{Background}

The progressive impairment of endothelial function and integrity starts a cascade of events, leading to microcirculation damage, atherosclerosis and common cardiovascular disease (CVD), such as coronary heart disease (CHD), myocardial infarction (MI), heart failure, stroke and peripheral arterial disease (PAD) [1]. Blood-derived endothelial progenitor cells (EPCs) represent the "promoters" of vascular repair providing the rationale for autologous stem cell therapy [2]. The coexistence of multiple classical CVD risk factors negatively influences the number and functional activity of EPCs [3,4]. The number of EPCs has been reported to negatively

\footnotetext{
* Correspondence: hwwang@ym.edu.tw

${ }^{2}$ Institute of Microbiology and Immunology, National Yang-Ming University, Taipei, Taiwan

${ }^{3}$ Institute of Biomedical Informatics, National Yang-Ming University, No. 155, Sec 2, Li-Nong Street, Taipei, Taiwan

Full list of author information is available at the end of the article
}

correlate with hypertension, diabetes mellitus and aging but not smoking [5]. Levels of EPCs are inversely correlated to progression of coronary heart disease [6]. EPCs are currently being tested in different clinical settings including repair of damaged microcirculation, regeneration of ischemic tissues, and bioengineering of vascular grafts (www.clinicaltrials.gov/).

Clinically, circulating EPCs can be obtained from adult peripheral blood and umbilical cord blood. The number of EPCs in adult blood is known to be significantly lower than in cord blood [7]. EPCs derived from different anatomic locations, just like other somatic stem cells of different sources $[8,9]$, possess unique biological activities: in vitro phenotypic studies demonstrated that CB-EPCs have competitive advantage compared with PB-EPCs due to their higher proliferative advantage, as well as better survival rate upon stress-induced apoptosis $[10,11]$. In vivo, tissue-engineered blood vessels generated by peripheral blood- and umbilical cord blood-

\section{Biomed Central}


derived EPCs: blood vessels formed by adult peripheral blood EPCs are unstable and regress within weeks, while umbilical cord blood EPCs form normal-functioning blood vessels that last for more than 4 months $[11,12]$. Thus, umbilical cord blood EPCs hold great therapeutic potential for cell therapy and vascular engineering.

The above findings suggest that CB-EPCs have enhanced vasculogenic ability compared with adult PB-EPCs. However, the underlying mechanisms are unclear. EPCs from human umbilical cord and adult peripheral blood activate different mechanisms upon high-dose $\mathrm{x}$-ray radiation treatment: CB-EPCs undergo p53 stabilization, Bax-dependent apoptosis and p21-dependent $\mathrm{G}_{1}$ and $\mathrm{G}_{2} / \mathrm{M}$ cell cycle checkpoints, while PB-EPCs undergo only radiation-induced senescence [13], indicating unique gene expression patterns in EPCs of different sources. Another level of regulation may lie on microRNAs (miRNAs), which are endogenously expressed small noncoding RNAs of 18-24 nucleotides in length that regulate gene expression on the posttranscriptional level [14]. microRNAs have emerged as master regulators of stem cell lineage differentiation and angiogenesis [14]. microRNAs also play a crucial role in endothelial inflammation, senescence and susceptibility to atherosclerosis: endothelial inflammation is critically regulated by miRNAs such as miR-126 and miR-10a, and endothelial aging is additionally controlled by miR-217 and miR-34a [15]. miR-221 and miR-222, which are encoded from the same miRNA cluster, modulate the angiogenic properties of human umbilical vein endothelial cells (HUVECs) by targeting $\mathrm{c}-\mathrm{Kit}$ and endothelial nitric oxide synthase (eNOS) [16]. In contrast, miRNA-31 enhances endothelial cell migration and invasion by targeting FAT4, a novel breast cancer tumor suppressor [17,18]. miR-126, -132, $-296,-378$, and the miR-17 92 cluster (encoding miR-17, $-18 \mathrm{a},-19 \mathrm{a} / \mathrm{b},-20 \mathrm{a}$ and $\mathrm{miR}-92 \mathrm{a})$ also contribute to pathological angiogenesis [19-21].

In this study we explored protein-coding mRNAs and miRNAs involved in EPC activities. We found that CBEPCs migrate faster and form tubule structures in vitro more efficiently than PB-EPCs do. mRNA and miRNA levels in EPCs of different origins reflect their unique performance.

\section{Methods}

\section{Isolation and cultivation of EPCs}

All patients gave informed consent, and the study was approved by the research ethics committee of the Hsinchu Mackay Memorial Hospital, Taiwan (ref number: 11MMHIS040). Protocols of this study were consistent with ethical guidelines provided in the 1975 Helsinki Declaration (http://www.wma.net/e/policy/b3. $\mathrm{htm})$. EPC isolation and characterization were done as described previously with minor modifications $[22,23]$.
In brief, blood mononuclear cells (MNCs) isolated by Histopaque-1077 (1.077 g/ml, Sigma, St. Louis, Missouri, USA) density-gradient centrifugation. MNCs $\left(1 \times 10^{7}\right)$ were plated in $2 \mathrm{ml}$ endothelial growth medium-2 (Lonza Ltd., Basel, Switzerland), with supplementation (hydrocortisone, IGF-1, human EGF, human VEGF, human FGF-B, ascorbic acid, GA-1000, heparin and 2\% fetal bovine serum) on fibronectin-coated six-well plates at $37{ }^{\circ} \mathrm{C}$ in a $5 \% \mathrm{CO}_{2}$ incubator. After 3 days of culturing, nonadherent cells were removed. Thereafter, the medium were replaced every 2 days, and EPCs colonies emerge 2-4 weeks after the start of MNC culture.

\section{EPC tube formation, transwell cell migration and cell proliferation assays}

A miR-31 cDNA construct was used in overexpression experiments [18]. Tube formation assay was performed on EPCs to assess their capacity for vasculogenesis, which is believed to be important in new vessel formation. In brief, the in vitro tube formation assay was performed by thawing Matrigel at $4{ }^{\circ} \mathrm{C}$ overnight, and then placed it in a 96-well plate at $37{ }^{\circ} \mathrm{C}$ for $1 \mathrm{~h}$ to allow the matrix solution to solidify. EPCs were harvested with trypsin/EDTA, and $1 \times 10^{4}$ EPCs were placed on Matrigel with EGM-2 medium or serum-free DMEM and incubated at $37{ }^{\circ} \mathrm{C}$ for $6 \mathrm{~h}$. Tubule formation was inspected under an inverted light microscope (100x). Four representative fields were taken. For 3D angiogenesis assay, collagen type I acidic solution were mixed with $1 / 2$ volume of basic conditioned medium with 0.2 ug/ml SDF- $1 \alpha$ (R\&D system, Minneapolis, MN USA) and solidify 30 minutes in 96 -well plate at $37{ }^{\circ} \mathrm{C}$ in a $5 \%$ $\mathrm{CO}_{2}$ incubator. $10^{5}$ cells per well were seeded and assayed.

Cell migration ability was evaluated using Costar Transwell ${ }^{\circledR}$ Polycarbonate Permeable Supports (Corning, NY, USA) as previously described [18]. The degree of cell proliferation was examined by the MTT assay system (Invitrogen, USA) according to the manufacturer's instructions.

\section{mRNA microarray and bioinformatics analysis}

Total RNA sample preparation, cRNA probe preparation, array hybridization and data analysis were done as described previously [24]. Affymetrix ${ }^{\mathrm{TM}}$ HG-U133 Plus 2.0 whole genome chips were used. RMA log expression units were calculated from Affymetrix GeneChip array data using the 'affy' package of the Bioconductor (http:// www.bioconductor.org) suite of software for the R statistical programming language (http://www.r-project.org). The default RMA settings were used to background correct, normalize and summarize all expression values. Significant differences between the sample groups was identified using the 'limma' (Linear Models for 
Microarray Analysis) package of the Bioconductor suite, and an empirical Bayesian moderated t-statistic hypothesis test between the two specified phenotypic groups was performed [25]. To control for multiple testing errors, we then applied a false discovery rate algorithm to these $p$ values in order to calculate a set of $q$ values, thresholds of the expected proportion of false positives, or false rejections of the null hypothesis [26]. Heat maps were created by the dChip software (http://www.dchip. org/). Array data are deposited in the Gene Expression Omnibus (GEO) database with an accession number of GSE39763. Part of the PB EPC array data were from a public GEO dataset GSE23203 (GSM663476-81) and 1 CB-EPC data from GSE12891 (GSM323169).

Gene annotation was performed by our ArrayFusion web tool (http://microarray.ym.edu.tw/tools/arrayfusion/ ) [27]. Gene Ontology database search were performed by the DAVID 6.7 Bioinformatics Resources (http:// david.abcc.ncifcrf.gov/). The Ingenuity Pathway Analysis (IPA) web tool developed by Ingenuity Co. (http://www. ingenuity.com) was used to construct functional regulatory networks of gene profiles. IPA uses the Ingenuity Pathways Knowledge Base to identify known interactions between focus genes and other genes that are not in the gene list. IPA then determines a statistical score for each network according to the fit of the network to the set of focus genes. The score is the negative $\log$ of $\mathrm{p}$ and denotes the likelihood of the focus genes in the network being found together by chance.

\section{Small RNA sequencing (smRNA-Seq) and data analysis}

Total RNA was collected and small RNA fractions were sequenced by Illumina Solexa Genome Analyzer IIx (GAIIx; Illumina, San Diego, CA USA) according to manufacturer's instruction. For data analysis, quality Fastq sequences, which were without poly-A, ambiguous nucleotides or a 5' adapter, yet flanking 6-18 nt of 3' adapter sequence, had the adapter sequences trimmed and the identical sequences were then collapse to unique sequences. The resulting unique sequences that did not align to mRNA database (UCSC genome browsers) but were aligned to known microRNA sequences (miRBase R18; http://www.mirbase.org/) were subjected into further quantification analysis. Sequencing reads were calculated to obtain a RPKM (reads per kilobase of exon model per million mapped reads) [28] value as C/LMN $\mathrm{x} 10^{9}$, where $\mathrm{C}=$ read numbers aligned to given miRNA chromosomal region, $\mathrm{L}=$ length of miRNA, $\mathrm{M}=$ multiple mapping numbers across all miRNA regions and $\mathrm{N}$ $=$ total read numbers that map to human genome sequence. microRNA target prediction was done by the miRTar webtool (http://mirtar.mbc.nctu.edu.tw/human/) [29].

\section{RNA extraction and real-time quantitative polymerase chain reaction (qPCR)}

RNA extraction and reverse transcription were performed as previously described [18]. The expression of mature human miRNAs was determined by a stem-loop real-time PCR system using the appropriate primer pairs. The universal PCR reverse primer for the miRNAs was 5'GTGCAGGGTCCGAGGT-3'. miR-31-specific primers were used [18] and the primer sequences are in Additional file 1: Figure S1. Primer sequences of all other genes and miRNAs are also in Additional file 1: Figure S1. The miRNA expression data were normalized against the average values of U6 snRNA, U48 snRNA and 5S rRNA, while the miRNA expression data were normalized against the average values of GAPDH and beta-actin.

\section{Results}

Isolation and characterization of human EPCs from cord blood and adult peripheral blood

EPCs were obtained from cord blood or peripheral blood of healthy subjects as described [23]. Blood MNCs that were initially seeded on fibronectin-coated wells were round, and outgrowth EPCs with a cobblestone-like morphology similar to mature endothelial cells grew to confluence at days 14-21 (not shown). Cultured EPCs were subjected into Traswell cell migration assays (Figure 1A), tube formation assays (Figure 1B), or MTT assays (Figure $1 C$ ). Clearly EPCs from CB migrated faster, proliferated faster and formed microvasculature structure more efficient in vitro (Figure 1A-C).

\section{Gene expression signatures and functional modules of different EPCs}

To provide the underlying mechanisms for observed phenotypes, we explored the transcriptome patterns of different EPCs. Protein-coding mRNAs were deciphered first by Affymetrix whole-genome microarrays. A total of 753 probe sets (positive false discovery rate (pFDR) q < 0.005 ) were found unique to CB-EPC, while another 431 to PB-EPC (Figure 2A \& Additional file 2: Figure S2 online). A PCA plot using these 1184 probe represents their differentiating power (Figure 2B).

The above gene list gave us a primary insight into the unique composition of differential EPCs but reflected little on EPC functions. To understand more how gene expression profiles might correlate with EPC biology and to provide quantitative evidence, signature probe sets were subjected to Gene Ontology (GO) database search for finding statistically over-represented functional groups within these genes. Given that the whole human transcriptome was represented by the microarray analysis, this analysis was not biased toward the coverage of the microarray. The GO categories of the biological processes being statistically overrepresented $(p<0.05)$ 


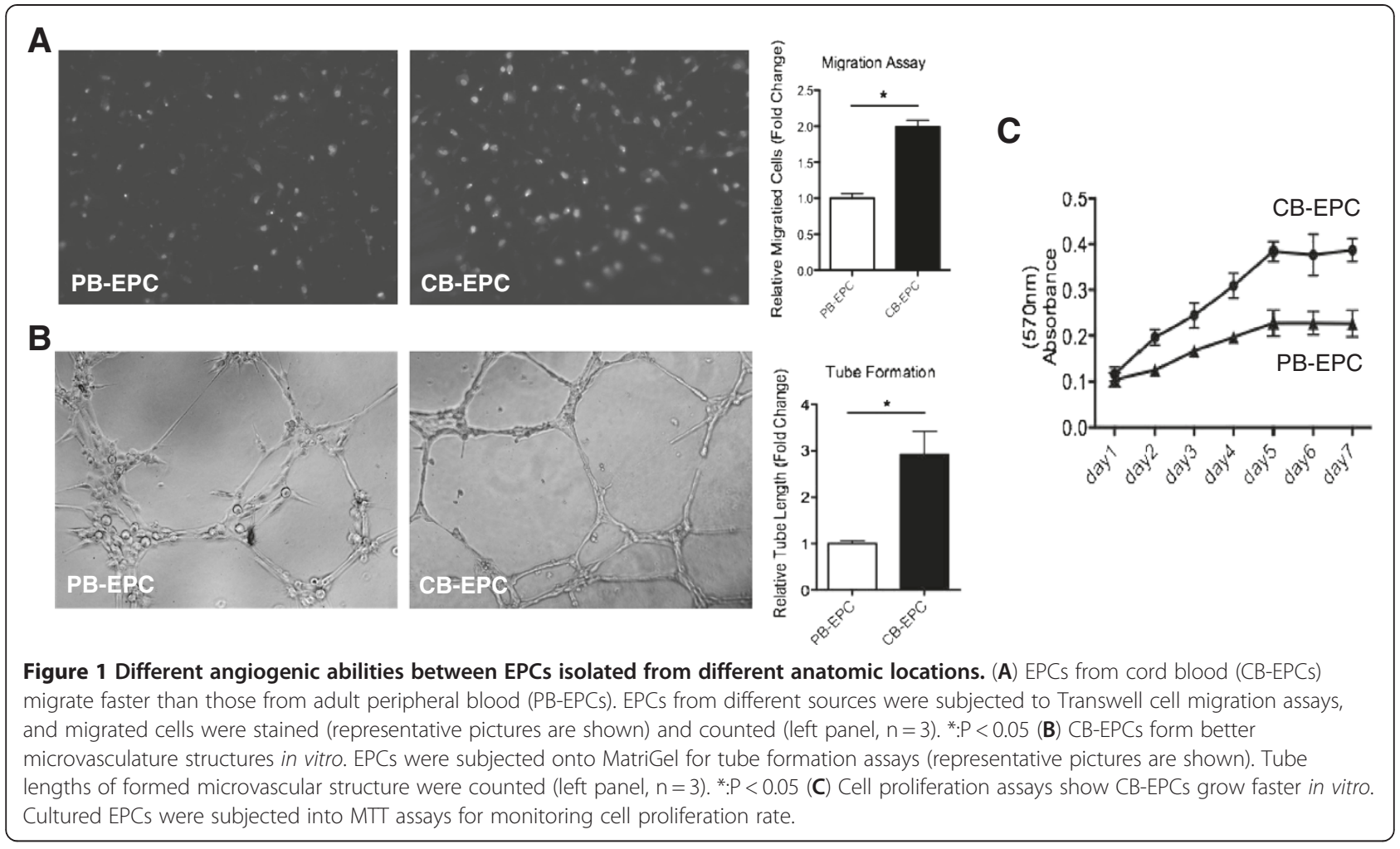

among CB-EPC genes are presented in Figure 2C. The most significant biological process for CB-EPCs is cell cycle (349 genes, $\left.p=1.14 \times 10^{-3}\right)$, especially the mitotic cell cycle (19 genes, $p=1.41 \times 10^{-4}$; Figure $\left.2 \mathrm{C}\right)$. Vasculature development genes (16 genes, $p=1.01 \times 10^{-2}$ ), especially those involved in angiogenesis (10 genes, $p=$ $3.73 \times 10^{-2}$ ), are also significantly higher in CB-EPCs (Figure 2C). The abundant expression of CB-EPC or PBEPC genes were verified by RT-qPCR (Figure 2D). A famous tumor suppressor TP53 was more abundant in PB-EPC, while angiogenic genes ANGPTL4 and CDK1 in CB-EPCs (Figure 2D). Other related predominant processes include those pertaining to DNA damage checkpoint ( 8 genes, $p=5380 \times 10^{-4}$, not shown), protein transport (38 genes, $p=0.0034$ ), and posttranslational protein modification (53 genes, $p=0.0045$, especially those involve in phosphorylation (37 genes, $p=0.0124)$ ).

We also subjected CB-EPC genes into KEGG and Ingenuity Pathway Analysis (IPA) database search for disclosing enriched pathways and functional modules. More information was revealed from Ingenuity database search. The "G2/M DNA damage checkpoint regulation" canonical pathway ranks the No. 1 most significant pathway found among CB-EPC genes (Figure 2E). Genes involved in the "FLT3 signaling in hematopoietic progenitor cells" pathway is also overexpresssed in CB-EPCs (Figures 2E-F). Also enriched in CB-EPCs are HIF1 $\alpha$ (hypoxia-inducible transcription factor 1 alpha) signaling, cardiac hypertrophy signaling, renin-angiotensin signaling and NFAT in cardiac hypertrophy pathways (Figure 2E), reflecting the pro-angiogenic nature of $\mathrm{CB}$ EPCs. By KEGG definition, genes involved in cell cycle are again found significant (Additional file 3: Figure S3). Database search and functional module assays explain in part why CB-EPCs amplification quicker (Figure 1C).

\section{Unique miRNA expression profiles of different EPCs revealed by small RNA sequencing (smRNA-Seq)}

Another level of gene expression regulation is through microRNAs. To provide a more comprehensive view of transcriptome profiles of EPCs from different sources, we determined miRNA profiles of different EPCs by sequencing the small RNA fractions of both EPCs. Illumina Solexa platform generated 9.7 million high-quality sequence reads for PB-EPCs, and another 11 million reads for CB-EPCs (Figure 3A, upper). We constructed an in-house pipeline (illustrated in Figure 3A) for analyzing sequencing data. The initial operations included identifying sequence matches to the mRNA database in order to eliminate degraded mRNA exon reads. Then non-exonic reads that match previously annotated miRNAs deposited in the miRBase database (release 18) were subjected to normalization and quantitative profiling. The expression of known miRNAs were converted into RPKM, and then filtered using a threshold RPKM > 


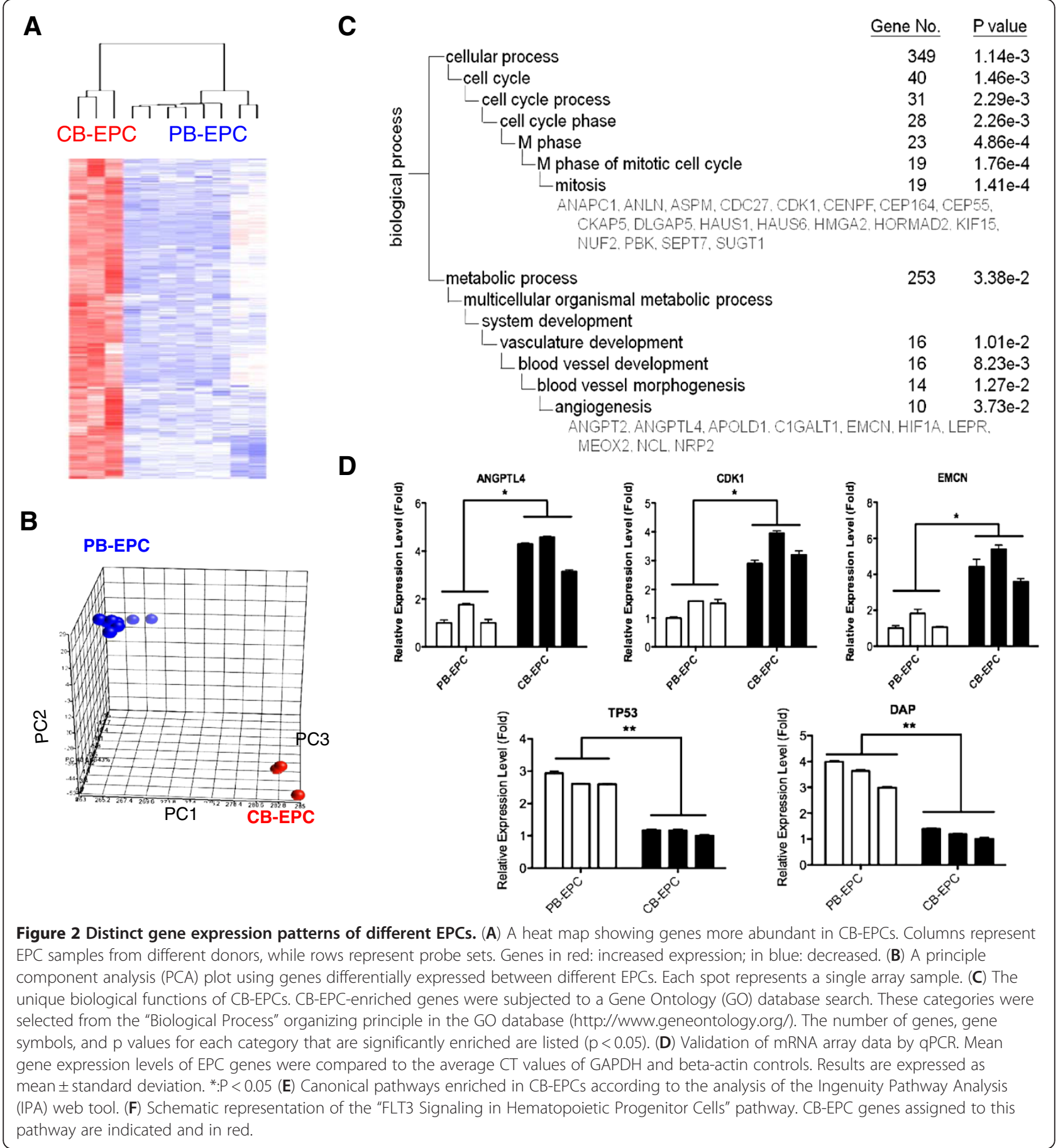

100. A total of 104 miRNAs were differentially expressed between $2 \mathrm{EPCs}$, with 54 being more abundant in CBEPCs while another 50 in PB-EPCs $(\geq 1.5$ folds; Figure 3B \& Tables 1-2). The differential expression of miR-31, miR-18a, miR-10a and miR-26a were verified by RT-qPCR (Figures 3C-D).

Applying the genetic network analysis function in the IPA web tool, we searched for miRNA-mRNA pairs and networks in CB-EPCs. PB-EPC miRNAs, such as miR- 10a/b, miR-26a, miR-103a, miR-107, miR-139-5p, miR151b, miR-361-5p, miR-365 and miR-1290, were found to be master regulators of a subset of CB-EPC proteincoding mRNAs (Figure 3E). The collective reduction of these miRNAs in CB-EPCs may explain in part why genes, such as ETV1 (Figure 3E), in this genetic network are more abundant in CB-EPCs.

Most of the differentially expressed miRNAs have not been linked to angiogenesis. MiR-410 is involved in 


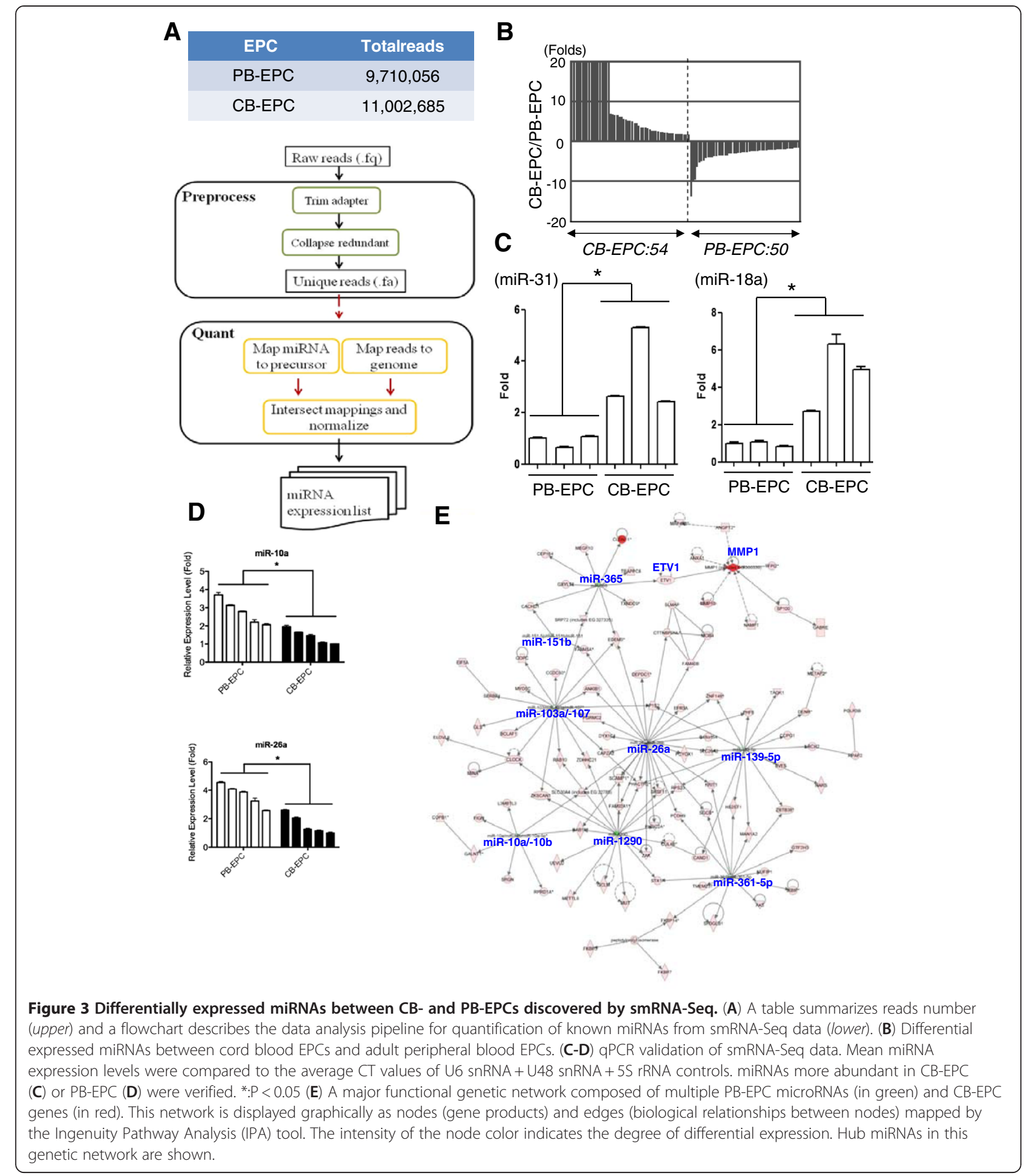

regulating secretion [30]. MiR-15a, -20b and -24 are reduced in the plasma of type 2 diabetes patients, which intend to have poor angiogenesis [31]. In vitro, miR-503 expression in ECs is upregulated in culture conditions mimicking diabetes mellitus (high $\mathrm{D}$-glucose) and ischemia-associated starvation (low growth factors).
Forced miR-503 expression inhibits EC proliferation, migration, and network formation on Matrigel [32]. MiR24 is considerably upregulated after cardiac ischemia and is enriched in cardiac endothelial cells. MiR-24 induces endothelial cell apoptosis, abolishes endothelial capillary network formation on Matrigel, and inhibits 
Table 154 miRNAs over-expressed in CB-EPC

\begin{tabular}{|c|c|c|c|c|c|c|}
\hline Name & chromosome location & PB RPKM & CB RPKM & Fold (CB/PB) & PB rank & CB rank \\
\hline hsa-mir-136 & chr14:101351053-101351075 & 0 & 495.64316 & $9.90 E+307$ & 770 & 95 \\
\hline hsa-mir-376c & chr14:101506069-101506089 & 1.2448007 & 1174.4226 & 943.4623551 & 483 & 68 \\
\hline hsa-mir-494 & chr14:101496018-101496039 & 3.3418648 & 3083.2217 & 922.6051575 & 382 & 33 \\
\hline hsa-mir-376a* & chr14:101507125-101507146 & 3.1190746 & 2104.9521 & 674.8643011 & 389 & 47 \\
\hline \multirow[t]{2}{*}{ hsa-mir-376a } & chr14:101506455-101506475 & 0.7002004 & 359.68243 & 513.6849822 & 542 & 106 \\
\hline & chr14:101507162-101507182 & & & & & \\
\hline hsa-mir-377 & chr14:101528431-101528452 & 0.89116406 & 305.87003 & 343.2252755 & 514 & 116 \\
\hline hsa-mir-410 & chr14:101532298-101532318 & 1.4004008 & 399.5301 & 285.2969664 & 468 & 101 \\
\hline hsa-mir-381 & chr14:101512305-101512326 & 6.6837296 & 1888.779 & 282.5935687 & 316 & 54 \\
\hline hsa-mir-411 & chr14:101489677-101489697 & 5.134803 & 1427.5367 & 278.0119705 & 341 & 63 \\
\hline hsa-mir-889 & chr14:101514286-101514306 & 1.4004008 & 309.84427 & 221.2539939 & 468 & 113 \\
\hline hsa-mir-379 & chr14:101488408-101488428 & 27.541208 & 4422.998 & 160.5956427 & 221 & 27 \\
\hline hsa-mir-369-3p & chr14:101531978-101531998 & 5.6016035 & 868.6908 & 155.0789519 & 334 & 81 \\
\hline hsa-mir-134 & chr14:101521031-101521052 & 10.9167595 & 910.0818 & 83.36556283 & 281 & 77 \\
\hline \multirow[t]{2}{*}{ hsa-mir-29b } & chr7:130562226-130562248 & 84.74453 & 6061.3887 & 71.52542707 & 162 & 20 \\
\hline & chr1:207975795-207975817 & & & & & \\
\hline hsa-mir-222* & chrX:45606479-45606500 & 25.843756 & 1562.2592 & 60.45016057 & 223 & 61 \\
\hline hsa-mir-31 & chr9:21512157-21512177 & 347.53278 & 15717.314 & 45.22541442 & 93 & 7 \\
\hline hsa-mir-127-3p & chr14:101349372-101349393 & 64.60941 & 2614.9524 & 40.47324376 & 174 & 39 \\
\hline hsa-mir-654-3p & chr14:101506606-101506627 & 16.932117 & 517.527 & 30.5648136 & 255 & 91 \\
\hline hsa-mir-146a & chr5:159912379-159912400 & 1061.7109 & 7229.664 & 6.809446903 & 65 & 15 \\
\hline hsa-mir-216a & chr2:56216155-56216176 & 462.73697 & 3054.6147 & 6.601190089 & 84 & 34 \\
\hline hsa-mir-18b & chrX:133304114-133304136 & 95.57735 & 621.04553 & 6.497831652 & 152 & 90 \\
\hline hsa-mir-24-2* & chr19:13947140-13947161 & 126.99086 & 810.7064 & 6.383974406 & 144 & 84 \\
\hline hsa-mir-503 & chrX:133680401-133680423 & 83.323845 & 498.72934 & 5.985433581 & 163 & 94 \\
\hline hsa-mir-18a & chr13:92003010-92003032 & 116.24848 & 684.41486 & 5.887516637 & 147 & 87 \\
\hline hsa-mir-4792 & chr3:24562903-24562920 & 56.91072 & 308.37952 & 5.418654341 & 181 & 114 \\
\hline hsa-mir-19a & chr13:92003193-92003215 & 168.31697 & 886.21814 & 5.265174034 & 130 & 80 \\
\hline \multirow[t]{2}{*}{ hsa-mir-19b } & chrX:133303713-133303735, & 351.0184 & 1759.4749 & 5.01248624 & 92 & 58 \\
\hline & chr13:92003499-92003521 & & & & & \\
\hline hsa-mir-185 & chr22:20020676-20020697 & 130.77832 & 639.70306 & 4.891506941 & 142 & 89 \\
\hline hsa-mir-424 & chrX:133680710-133680731 & 326.61172 & 1557.3126 & 4.768085481 & 97 & 62 \\
\hline \multirow[t]{2}{*}{ hsa-mir-24 } & chr9:97848346-97848367 & 39987.26 & 172634.36 & 4.317234039 & 5 & 2 \\
\hline & chr19:13947103-13947124 & & & & & \\
\hline \multirow[t]{2}{*}{ hsa-mir-196a } & chr17:46709894-46709915 & 93.497955 & 398.9361 & 4.266789578 & 156 & 102 \\
\hline & chr12:54385546-54385567 & & & & & \\
\hline hsa-mir-130a & chr11:57408725-57408746 & 319.70517 & 1143.3563 & 3.576283424 & 98 & 70 \\
\hline hsa-mir-345 & chr14:100774213-100774234 & 137.23924 & 455.79385 & 3.321162737 & 138 & 98 \\
\hline hsa-mir-32 & chr9:111808552-111808573 & 94.68617 & 312.1079 & 3.296235343 & 153 & 112 \\
\hline hsa-mir-339-3p & chr7:1062591-1062613 & 116.35501 & 376.72174 & 3.237692472 & 146 & 105 \\
\hline hsa-mir-186 & chr1:71533364-71533385 & 1265.8989 & 3930.9248 & 3.105243871 & 57 & 30 \\
\hline hsa-mir-20b & chrX:133303880-133303902 & 393.07117 & 1119.9288 & 2.849175634 & 90 & 72 \\
\hline hsa-mir-542-3p & chrX:133675394-133675415 & 302.77307 & 758.6524 & 2.505679914 & 100 & 85 \\
\hline hsa-mir-877 & chr6:30552109-30552128 & 134.05331 & 333.61777 & 2.488694759 & 140 & 110 \\
\hline
\end{tabular}


Table 154 miRNAs over-expressed in CB-EPC (Continued)

\begin{tabular}{|c|c|c|c|c|c|c|}
\hline hsa-mir-106b & chr7:99691666-99691686 & 1116.7422 & 2731.2852 & 2.445761609 & 62 & 36 \\
\hline hsa-mir-452 & chrX:151128150-151128171 & 466.9701 & 1105.1763 & 2.366696069 & 82 & 73 \\
\hline hsa-mir-22 & chr17:1617208-1617229 & 1037.9835 & 2326.5046 & 2.24136954 & 66 & 42 \\
\hline hsa-mir-374a* & chrX:73507130-73507151 & 1247.1844 & 2619.4692 & 2.100306258 & 60 & 38 \\
\hline hsa-mir-29c & chr1:207975210-207975231 & 1326.5715 & 2675.448 & 2.01681402 & 53 & 37 \\
\hline hsa-mir-29a & chr7:130561507-130561528 & 1443.6847 & 2802.1428 & 1.940965919 & 50 & 35 \\
\hline hsa-mir-30e & chr1:41220043-41220064 & 5007.227 & 9227.503 & 1.842836963 & 26 & 10 \\
\hline hsa-mir-99a & chr21:17911421-17911442 & 2773.0786 & 5025.056 & 1.812085673 & 41 & 24 \\
\hline hsa-mir-20a & chr13:92003326-92003348 & 1287.8253 & 2302.079 & 1.787570876 & 54 & 43 \\
\hline hsa-mir-15a & chr13:50623303-50623324 & 483.79068 & 822.4292 & 1.699969086 & 81 & 83 \\
\hline hsa-mir-100 & chr11:122022983-122023004 & 4361.801 & 7380.455 & 1.692065961 & 28 & 14 \\
\hline hsa-mir-106a & chrX:133304274-133304296 & 1250.3193 & 2036.8033 & 1.629026521 & 59 & 50 \\
\hline hsa-mir-17 & chr13:92002872-92002894 & 1266.1599 & 2058.4065 & 1.625708175 & 56 & 48 \\
\hline hsa-mir-27a & chr19:13947261-13947281 & 5321.171 & 8077.8022 & 1.51804973 & 25 & 12 \\
\hline hsa-mir-140-5p & chr16:69967006-69967027 & 334.1866 & 501.82477 & 1.501630436 & 96 & 93 \\
\hline
\end{tabular}

cell sprouting from endothelial spheroids by targeting of the stemness transcription factor GATA2 and the p21-activated kinase PAK4 [33,34]. MiR-100 has an antiangiogenic function by modulating proliferation, tube formation, and sprouting activity of endothelial cells and migration of vascular smooth muscle cells and functions as an endogenous repressor of the serine/threonine protein kinase mammalian target of rapamycin (mTOR) [35]. MiR-29b can suppress tumor angiogenesis, invasion and metastasis by regulating MMP-2 expression in hepatocellular carcinoma (HCC) [36]. MicroRNAs from the miR-17 92 cluster are known to contribute in pathological angiogenesis [19-21], and 5 out of 6 members (including miR-17, $-18 \mathrm{a},-19 \mathrm{a} / \mathrm{b}$ and $-20 \mathrm{a})$ were overexpressed in CB-EPCs (Table 1).

\section{miR-31 as a novel EPC angiogenic miRNA}

To identify more pro-angiogenic miRNAs involved in EPC activity, we examined which miRNA(s) may contribute in EPC angiogenesis. MiR-31 is a known proangiogenic and pro-lymphangiogenic miRNA which induce motility in both matured blood vessel and lymphatic endothelial cells [18,37]. Knocking down endogenous miR-31 levels reduced tube formation and cellular migration abilities in CB-EPCs (Figures 4A-C), suggesting a pro-angiogenic role of $\mathrm{miR}-31$ in both progenitor and mature type endothelial lineage cells. On the other hand, overexpressing miR-31 in PB-EPCs helped to recapitulate some of the functions of CB-EPCs (Figure 4D). Over-expressing miR-31 in PB-EPC or knocking down endogenous CB-EPC miR-31 level did not affect cell proliferation rate at a significant level in the first 24 hours of transfection (Additional file 4:
Figure S4). The tube formation and cell migration effects we observed should due to mainly the pro-angiogenic activity of miR-31.

\section{Discussion}

Endothelial cells from the internal barrier of the vasculature, and play fundamental roles in vascular development and disease. The regulation of angiogenesis depends not only on the number of circulating EPC but also on their functions [38]. Aberrant EPC activity and the resulting abnormal angiogenesis cause a variety of diseases, such as ischemia, cancer and metastasis. On the other hand, these cells are also potential cell source for cellular therapies aiming to enhance the neovascularization of tissue engineered constructs or ischemic tissues [39]. Atherosclerotic heart disease remains one of the major causes of morbidity and mortality worldwide. Currently, vascular revascularization techniques, including balloon angioplasty and stenting, have been well developed. However, post-angioplasty restenosis substantially limit long-term benefits of heart revascularization procedures. Therapeutic progenitor cell transplantation bear potential for organ vascularization regeneration in various pathological states [40]. The application of EPC in stenting technology during vascular revascularization is the Genous EPC stent (OrbusNeich, Wanchai, Hong Kong), which is a stainless-steel stent coated with immobilized human anti-CD34 monoclonal antibodies that allow the stent surface to "capture" EPCs in the blood to accelerate endothelialization of the stent strut. In recent clinical trial, it shows promise result in reducing the risk of stent thrombosis by facilitating rapid endothelialization on stent strut [41]. 
Table 250 miRNAs over-expressed in PB-EPC

\begin{tabular}{|c|c|c|c|c|c|c|}
\hline Name & chromosome location & PB RPKM & CB RPKM & Fold (CB/PB) & PB rank & CB rank \\
\hline hsa-let-7b* & chr22:46509625-46509646 & 358.35938 & 26.206163 & -13.67462226 & 91 & 280 \\
\hline hsa-mir-15b* & chr3:160122433-160122454 & 695.9995 & 72.2731 & -9.630132096 & 73 & 215 \\
\hline hsa-mir-1290 & chr1:19223572-19223590 & 337.0359 & 35.74031 & -9.43013365 & 95 & 258 \\
\hline hsa-mir-15b & chr3:160122395-160122416 & 2379.7402 & 379.11118 & -6.27715648 & 42 & 104 \\
\hline hsa-mir-574-3p & chr4:38869713-38869734 & 435.55652 & 86.03938 & -5.062292639 & 85 & 201 \\
\hline hsa-mir-25 & chr7:99691194-99691215 & 1534.8059 & 319.06284 & -4.810356167 & 49 & 111 \\
\hline hsa-mir-148a & chr7:25989542-25989563 & 1073.8528 & 231.33844 & -4.641912516 & 63 & 134 \\
\hline hsa-mir-30e* & chr1:41220085-41220106 & 3634.1675 & 920.4779 & -3.948131183 & 34 & 76 \\
\hline hsa-mir-30a* & chr6:72113257-72113278 & 4051.6772 & 1061.4753 & -3.817024475 & 29 & 74 \\
\hline \multirow[t]{2}{*}{ hsa-mir-365 } & chr16:14403197-14403218, & 653.44617 & 171.86374 & -3.802117713 & 75 & 149 \\
\hline & chr17:29902497-29902518 & & & & & \\
\hline hsa-mir-28-3p & chr3:188406622-188406643 & 1804.1597 & 485.26227 & -3.717906401 & 44 & 96 \\
\hline hsa-mir-23b & chr9:97847547-97847567 & 8076.4937 & 2225.3198 & -3.629363159 & 22 & 45 \\
\hline hsa-mir-146b-5p & chr10:104196277-104196298 & 1253.9794 & 349.96533 & -3.583153223 & 58 & 108 \\
\hline hsa-mir-92b & chr1:155165028-155165049 & 318.21985 & 90.19796 & -3.528016044 & 99 & 195 \\
\hline hsa-mir-197 & chr1:110141562-110141583 & 528.68317 & 149.92363 & -3.526349849 & 79 & 156 \\
\hline hsa-mir-140-3p & chr16:69967045-69967065 & 12527.033 & 3577.7405 & -3.501381109 & 12 & 32 \\
\hline hsa-mir-598 & chr8:10892731-10892752 & 489.91757 & 143.68575 & -3.40964619 & 80 & 161 \\
\hline hsa-let-7b & chr22:46509571-46509592 & 8703.284 & 2554.5288 & -3.407001714 & 20 & 40 \\
\hline hsa-mir-378 & chr5:149112430-149112450 & 32406.646 & 10804.972 & -2.999234612 & 7 & 9 \\
\hline hsa-mir-455-3p & chr9:116971767-116971787 & 548.25714 & 187.93459 & -2.917276378 & 78 & 142 \\
\hline hsa-mir-193b & chr16:14397874-14397895 & 415.28253 & 143.25558 & -2.898892525 & 87 & 162 \\
\hline \multirow[t]{2}{*}{ hsa-mir-92a } & chrX:133303574-133303595 & 3847.525 & 1331.7456 & -2.889084071 & 33 & 64 \\
\hline & chr13:92003615-92003636 & & & & & \\
\hline hsa-mir-93 & chr7:99691438-99691460 & 3588.8904 & 1322.95 & -2.712793681 & 35 & 65 \\
\hline hsa-let-7c & chr21:17912158-17912179 & 15608.78 & 5758.0083 & -2.710794981 & 11 & 22 \\
\hline hsa-mir-30b & chr8:135812813-135812834 & 1066.1298 & 400.58517 & -2.661431026 & 64 & 100 \\
\hline hsa-mir-10a & chr17:46657266-46657288 & 17177.37 & 6494.583 & -2.644876507 & 8 & 17 \\
\hline hsa-mir-10b & chr2:177015057-177015079 & 16707.91 & 6317.509 & -2.644699042 & 10 & 19 \\
\hline hsa-let-7d & chr9:96941123-96941144 & 9057.177 & 3642.2817 & -2.48667669 & 16 & 31 \\
\hline hsa-mir-151-3p & chr8:141742686-141742706 & 17066.672 & 7218.801 & -2.3641976 & 9 & 16 \\
\hline \multirow[t]{2}{*}{ hsa-mir-30c } & chr1:41222972-41222994 & 4914.325 & 2188.866 & -2.245146574 & 27 & 46 \\
\hline & chr6:72086706-72086728 & & & & & \\
\hline hsa-let-7e & chr19:52196046-52196067 & 8924.559 & 3985.3586 & -2.239336505 & 17 & 29 \\
\hline hsa-mir-320a & chr8:22102488-22102509 & 757.8811 & 340.61673 & -2.225026058 & 70 & 109 \\
\hline \multirow[t]{3}{*}{ hsa-let-7a } & chr11:122017276-122017297 & 65673.34 & 30296.24 & -2.16770596 & 3 & 5 \\
\hline & chr9:96938244-96938265 & & & & & \\
\hline & chr22:46508632-46508653 & & & & & \\
\hline hsa-mir-99b & chr19:52195871-52195892 & 8875.32 & 4099.9907 & -2.164717105 & 19 & 28 \\
\hline \multirow[t]{2}{*}{ hsa-let-7f } & chr9:96938635-96938656 & 37668.82 & 17587.143 & -2.141838501 & 6 & 6 \\
\hline & chrX:53584207-53584228 & & & & & \\
\hline hsa-mir-23a & chr19:13947409-13947429 & 10271.966 & 4879.2725 & -2.105224908 & 14 & 26 \\
\hline hsa-mir-125b & chr21:17962573-17962594 & 3983.9465 & 1976.1088 & -2.016056252 & 30 & 51 \\
\hline
\end{tabular}


Table 250 miRNAs over-expressed in PB-EPC (Continued)

\begin{tabular}{|c|c|c|c|c|c|c|}
\hline hsa-mir-125a-5p & chr19:52196521-52196544 & 1764.7084 & 897.535 & -1.966172238 & 46 & 79 \\
\hline hsa-mir-361-5p & chrX:85158686-85158707 & 399.24158 & 205.20401 & -1.945583714 & 89 & 138 \\
\hline \multirow[t]{2}{*}{ hsa-mir-16 } & chr3:160122542-160122563 & 3338.2983 & 1761.0107 & -1.895671787 & 38 & 57 \\
\hline & chr13:50623163-50623184 & & & & & \\
\hline hsa-mir-1307 & chr10:105154058-105154079 & 553.6359 & 294.46994 & -1.880110072 & 77 & 118 \\
\hline \multirow[t]{2}{*}{ hsa-mir-320b } & chr1:224444751-224444772 & 661.5061 & 356.97238 & -1.853101632 & 74 & 107 \\
\hline & chr1:117214409-117214430 & & & & & \\
\hline hsa-mir-217 & chr2:56210155-56210177 & 10865.332 & 5983.509 & -1.815879612 & 13 & 21 \\
\hline hsa-mir-769-5p & chr19:46522219-46522240 & 421.96628 & 236.17818 & -1.786643796 & 86 & 133 \\
\hline hsa-mir-191 & chr3:49058105-49058127 & 3378.5552 & 1927.2273 & -1.753065246 & 36 & 52 \\
\hline hsa-mir-139-5p & chr11:72326147-72326168 & 301.21347 & 178.96194 & -1.683114689 & 101 & 145 \\
\hline \multirow[t]{2}{*}{ hsa-mir-26a } & chr12:58218441-58218462 & 3344.9814 & 2055.193 & -1.627575318 & 37 & 49 \\
\hline & chr3:38010904-38010925 & & & & & \\
\hline \multirow[t]{2}{*}{ hsa-mir-103a } & chr5:167987909-167987931 & 8117.7095 & 5296.3853 & -1.532688624 & 21 & 23 \\
\hline & chr20:3898188-3898210 & & & & & \\
\hline hsa-mir-107 & chr10:91352513-91352535 & 3862.0547 & 2533.423 & -1.524441319 & 31 & 41 \\
\hline hsa-mir-151b & chr14:100575775-100575792 & 610.3607 & 405.608 & -1.504804392 & 76 & 99 \\
\hline
\end{tabular}

An increasing number of studies shows that miRNAs, or angiomiRs, play a crucial role in regulating various aspects of cancer biology, including angiogenesis. Manipulating miRNAs in the settings of pathological vascularization therefore represents a new therapeutic approach [14]. On the other hand, there are still challenges to harnessing EPCs for cell therapy. One of these is their rarity (0.01-0.02 per $10^{6}$ mononuclear cells), which makes EPC isolation challenging. In vitro cultivation and amplification of EPCs is therefore required before these cells may be appropriately investigated for use in clinical therapies. However, it is crucial to maintain EPC activity during such in vitro manipulation. Understanding the basic EPC biology will help to develop new biomarkers for monitoring EPC activities. In this report, we identified EPCs, especially those from cord blood, exploit several cellular genetic groups and miRNA pathways to regulate their angiogenesis activities. Transcriptome information will eventually help to develop new clinical applications as mentioned above.

When PB-EPC genes were subjected into GO database search, we found these genes are enriched in both Wnt receptor signaling (8 genes including CREBBP, DVL3, NFAT5, PPARD, RAC1, TBL1X, TCF7L2, TP53; $\mathrm{p}=0.019$ ) and positive regulation of I-kappaB kinase/NF-kappaB cascade (6 genes including LITAF, MAP3K3, MAP3K7IP2, MUL1, TRIM13, PSMB7; $\mathrm{p}=0.048$ ). Genes involved in the induction of apoptosis are also more abundant in PBEPCs (15 genes, $p=0.006)$. KEGG and IPA database search also revealed that genes involved in both Wnt signaling $(\mathrm{p}=0.020)$ and MAPK (13 genes, including
ACVR1B, ARRB1, DDIT3, DUSP3, DUSP16, ELK4, GADD45B, MAP3K3, MAP3K7IP1, MAP3K7IP2, MAPKAPK2, RAC1 and TP53; $p=012$ ) pathways are more abundant in PB-EPCs (Additional file 5: Figure S5, Additional file 6: Table S1). IPA analysis also revealed the Wnt/ $\beta$-catenin signaling pathway may be more active in PB-EPCs (Additional file 7: Table S2). The Wnt signaling system regulates vascular patterning in the developing embryo [42]. It has recently been documented that Wnt1 is a proangiogenic molecule of human endothelial progenitor function, and increases blood flow to ischemic limbs in a HGF-dependent manner [43]. Our work further supports a crucial role of Wnt pathway in adult EPCs, and Wnt proteins may be therapeutically deployed to increase blood flow and angiogenesis in adult ischemic tissues.

In this study we applied RNA sequencing (RNA-seq) technology, instead of microRNA chips, for deciphering EPC miRNomes. This is due to the fact that microarray application in miRNome research has several limitations, including hybridization and cross-hybridization artifacts, dye-based detection issues and design constraints that preclude or seriously limit the detection of newly discovered miRNAs or previously unmapped, novel miRNAs [44]. These issues have made it difficult for standard array designs to provide full sequence comprehensiveness (coverage of all possible genes, including "unknown" ones, in large genomes) or transcriptome comprehensiveness (reliable detection of all RNAs of all prevalence classes, including the least abundant ones that are physiologically relevant). Studies using this method have already altered our view of the extent and 


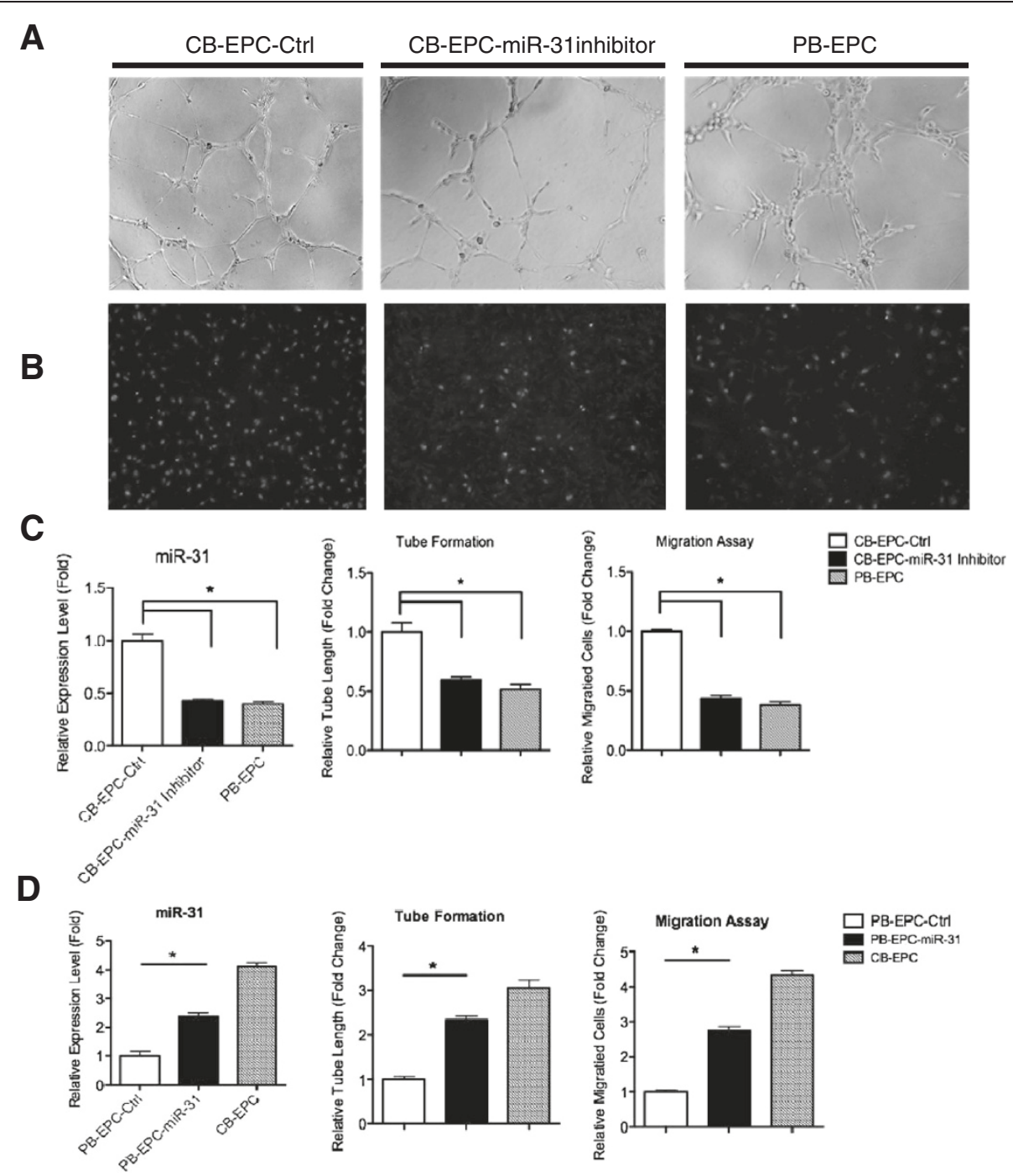

Figure 4 miR-31 is involved in EPC activities. (A-C) Knocking down endogenous miR-31 in CB-EPCs. Anti-miR-31 antagomiR or the siGFP control (Ctrl) was introduced into CB-EPCs by electroporation, and 2 days later EPCs were subjected to tube formation (A) and Transwell cell migration assays (B). Cellular miR-31 levels were detected by RT-qPCR ( $C$, left panel; $n=3)$, and migration assay and tube formation assay results were also quantified $(C$, middle and right panels; $n=3$, using cells from 3 batches of donors). (D) Overexpressing miR-31 in PB-EPCs stimulates EPC angiogenic abilities. Cellular miR-31 levels were detected by RT-qPCR (left panel; $n=3$ ). Cellular migration assays and tube formation assays were done, and results were quantified (middle and right panels; $n=3$, using cells from 3 batches of donors).

complexity of eukaryotic transcriptomes [44]. RNA-seq has also delivered a sharp rise in the rate of novel microRNA discovery in the current miRBase R18 release (2011 Nov; http://microrna.sanger.ac.uk/sequences/), which is the primary online repository for all microRNA sequences and annotation.

One of the CB-EPC-dominant microRNAs is miR-31, a pro-angiogenic miRNA that enhances endothelial cell migration [18,37]. MiR-31 has recently been documented as a signature BEC miRNA that negatively regulates lymphatic endothelial cell identity and lymphatic vascular development by targeting Prox1, a transcription factor that functions as a master regulator of lymphatic lineage-specific differentiation [45]. In the present study, we further showed that miR-31 is a dominant miRNA in CB-EPCs, and its overexpression is crucial for EPCs to possess superior angiogenic ability (Figure 4). Unmasking the roles of small RNA-mediated gene regulation in EPC activity will be crucial and will provide new insights into regenerative and reparative medicine. We envision that our report will serve as a resource for future miRNA studies that aim to improve understanding of the various regulatory ultimately modulating EPC and EC activities.

For miRNAs more abundant in PB-EPCs, miR-217 modulates endothelial cell senescence via silent information 
regulator 1 (sirT1) [46]. The levels of miR-330 and let-7e are higher in the myocardial microvascular endothelial cells (MMVEC) in type 2 diabetic Goto-Kakizaki (GK) rats, which have impaired angiogenesis [47]. MiR-93, member of the miR-106b $\sim 25$ cluster (a paralog of the miR-17 $\sim 92$ cluster), in tumor cells possesses oncogenic and angiogenic activities [48]. Both microRNA125a-5p and miR-125b are overexpressed in PB-EPCs (Table 2), and their role in inhibiting endothelin-1 expression in vascular endothelial cells has been reported [49]. miR-10a regulates a proinflammatory phenotype in athero-susceptible endothelium in vivo [50]. The collective effects of these miRNAs in EPC biology are still awaited to be elucidated.

\section{Conclusions}

In summary, our results reveal a series of differentially expressed miRNAs and protein-coding genes that have not previously been associated with EPC biology. This study therefore provides a road map for future mechanistic studies of EPC migration and microvasculature formation, which should eventually help to improve our understanding of angiogenesis, and will also benefit the development of new therapeutic approaches that target the inhibition of pathogenic angiogenesis in tumors, the stimulation of angiogenesis in patients with cardiovascular diseases, stroke or diabetes.

\section{Additional files}

\section{Additional file 1: Figure S1 Primers used in RT-qPCR validation. \\ Additional file 2: Figure S2 Gene expression signatures and functional modules of different EPCs.}

Additional file 3: Figure S3 Distribution of CB-EPC cell cycle genes according to the KEGG database. CB-EPC genes are labeled with red stars. The $P$ value is also shown.

Additional file 4: Figure S4 Over-expressing miR-31 in PB-EPC (A) or knocking down endogenous miR-31 in CB-EPC (B) did not affect cell proliferation rate at a significant level in the first 24 hours of transfection.

Additional file 5: Figure S5: Distribution of PB-EPC genes in the Wnt signaling pathway according to the KEGG database. PB-EPC genes are labeled with red stars.

Additional file 6: Table S1 Distribution of PB-EPC genes in the MAPK signaling pathway according to the KEGG database. PB-EPC genes are labeled with red stars.

Additional file 7: Table S2 Distribution of PB-EPC genes in the Wnt signaling pathway according to the IPA web tool. Involved PB-EPC genes are in green and indicated.

\section{Competing interests}

The authors declare no competing financial interests.

\section{Authors' contributions}

$\mathrm{C}-\mathrm{CC}$ and $\mathrm{H}-\mathrm{HL}$ carried out the study design, performed the statistical analysis and drafted the manuscript. $\mathrm{H}-\mathrm{H} \mathrm{L}$ and $\mathrm{Y}$-CCcarried out the cell biology assays. T-SH and S-TC participated in sequence alignment. S-JC provided clinical samples. H-WW conceived of the study, and participated in its design and coordination and helped to draft the manuscript. All authors read and approved the final manuscript.

\section{Acknowledgements}

The authors acknowledge the technical services provided by Microarray \& Gene Expression Analysis Core Facility of the National Yang-Ming University VGH Genome Research Center, which is supported by National Research

Program for Genomic Medicine, National Science Council (NSC). This work is supported by NSC (NSC101-2320-B-010-059-MY3, NSC98-2320-B-010-020-MY3 and NSC100-2627-B-010-007), Tri-Service General Hospital (TSGH-C102-027), the Yen Tjing Lin Medical Foundation (Cl-100-23), Mackay Memorial Hospital (MMH-HB-100-01), Taipei Veteran General Hospital (V101E2-008 and Cancer Excellence Center Plan DOH101-TD-C-111-007) and National Yang-Ming University (Ministry of Education, Aim for the Top University Plan). This work is also support in part by the UST-UCSD International Center for Excellence in Advanced Bioengineering sponsored by the Taiwan NSC I-RiCE Program under Grant Number: NSC100-2911-I-009-101.

\section{Author details}

${ }^{1}$ Division of Cardiology, Department of Internal Medicine, Tri-Service General Hospital, National Defense Medical Center, Taipei, Taiwan. ${ }^{2}$ Institute of Microbiology and Immunology, National Yang-Ming University, Taipei, Taiwan. ${ }^{3}$ Institute of Biomedical Informatics, National Yang-Ming University, No. 155, Sec 2, Li-Nong Street, Taipei, Taiwan. ${ }^{4}$ Department of Obstetrics and Gynecology, Hsinchu Mackay Memorial Hospital, Hsinchu, Taiwan.

${ }^{5}$ Department of Education and Medical Research, Taipei City Hospital, Taipei, Taiwan. ${ }^{6}$ National Yang-Ming University VGH Genome Research Center,

National Yang-Ming University, Taipei, Taiwan.

Received: 16 January 2012 Accepted: 14 August 2012

Published: 3 September 2012

\section{References}

1. Crimi E, Ignarro LJ, Napoli C: Microcirculation and oxidative stress. Free Radic Res 2007, 41(12):1364-1375.

2. Dzau VJ, Gnecchi M, Pachori AS, Morello F, Melo LG: Therapeutic potential of endothelial progenitor cells in cardiovascular diseases. Hypertension 2005, 46(1):7-18.

3. Kinnaird T, Stabile E, Zbinden S, Burnett MS, Epstein SE: Cardiovascular risk factors impair native collateral development and may impair efficacy of therapeutic interventions. Cardiovasc Res 2008, 78(2):257-264.

4. Feihl F, Liaudet L, Levy BI, Waeber B: Hypertension and microvascular remodelling. Cardiovasc Res 2008, 78(2):274-285.

5. Umemura T, Soga J, Hidaka T, Takemoto H, Nakamura S, Jitsuiki D, Nishioka K, Goto C, Teragawa H, Yoshizumi M, et al: Aging and hypertension are independent risk factors for reduced number of circulating endothelial progenitor cells. Am J Hypertens 2008, 21(11):1203-1209.

6. Briguori C, Testa U, Riccioni R, Colombo A, Petrucci E, Condorelli G, Mariani G, D'Andrea D, De Micco F, Rivera NV, et al: Correlations between progression of coronary artery disease and circulating endothelial progenitor cells. Faseb J 2010, 24(6):1981-1988.

7. Peichev M, Naiyer AJ, Pereira D, Zhu Z, Lane WJ, Williams M, Oz MC, Hicklin DJ, Witte L, Moore MA, et al: Expression of VEGFR-2 and AC133 by circulating human $\mathrm{CD} 34(+)$ cells identifies a population of functional endothelial precursors. Blood 2000, 95(3):952-958.

8. Hsieh JY, Fu YS, Chang SJ, Tsuang YH, Wang HW: Functional module analysis reveals differential osteogenic and stemness potentials in human mesenchymal stem cells from bone marrow and Wharton's jelly of umbilical cord. Stem Cells Dev 2010, 19(12):1895-1910.

9. Wang TY, Chang SJ, Chang MD, Wang HW: Unique biological properties and application potentials of CD34+ CD38- stem cells from various sources. Taiwan J Obstet Gynecol 2009, 48(4):356-369.

10. Ingram DA, Mead LE, Tanaka H, Meade V, Fenoglio A, Mortell K, Pollok K, Ferkowicz MJ, Gilley D, Yoder MC: Identification of a novel hierarchy of endothelial progenitor cells using human peripheral and umbilical cord blood. Blood 2004, 104(9):2752-2760.

11. Au P, Daheron LM, Duda DG, Cohen KS, Tyrrell JA, Lanning RM, Fukumura D, Scadden DT, Jain RK: Differential in vivo potential of endothelial progenitor cells from human umbilical cord blood and adult peripheral blood to form functional long-lasting vessels. Blood 2008, 111(3):1302-1305. 
12. Melero-Martin JM, Khan ZA, Picard A, Wu X, Paruchuri S, Bischoff J: In vivo vasculogenic potential of human blood-derived endothelial progenitor cells. Blood 2007, 109(11):4761-4768.

13. Mendonca MS, Chin-Sinex H, Dhaemers R, Mead LE, Yoder MC, Ingram DA Differential mechanisms of $x$-ray-induced cell death in human endothelial progenitor cells isolated from cord blood and adults. Radiat Res 2011, 176(2):208-216

14. Wang $\mathrm{S}$, Olson EN: AngiomiRs-key regulators of angiogenesis. Curr Opin Genet Dev 2009, 19(3):205-211.

15. Qin B, Yang $H$, Xiao B: Role of microRNAs in endothelial inflammation and senescence. Mol Biol Rep 2011, 39(4):4509-18.

16. Kuehbacher A, Urbich C, Dimmeler S: Targeting microRNA expression to regulate angiogenesis. Trends Pharmaco/ Sci 2008, 29(1):12-15.

17. Qi C, Zhu YT, Hu L, Zhu YJ: Identification of Fat4 as a candidate tumor suppressor gene in breast cancers. Int J Cancer 2009, 124(4):793-798.

18. Wu YH, Hu TF, Chen YC, Tsai YN, Tsai YH, Cheng CC, Wang HW: The manipulation of miRNA-gene regulatory networks by KSHV induces endothelial cell motility. Blood 2011, 118(10):2896-2905.

19. Lee DY, Deng Z, Wang CH, Yang BB: MicroRNA-378 promotes cell survival, tumor growth, and angiogenesis by targeting SuFu and Fus-1 expression. Proc Natl Acad Sci U S A 2007, 104(51):20350-20355.

20. Wurdinger T, Tannous BA, Saydam O, Skog J, Grau S, Soutschek J, Weissleder R, Breakefield XO, Krichevsky AM: miR-296 regulates growth factor receptor overexpression in angiogenic endothelial cells. Cancer Cell 2008, 14(5):382-393.

21. Anand S, Majeti BK, Acevedo LM, Murphy EA, Mukthavaram R, Scheppke L, Huang M, Shields DJ, Lindquist JN, Lapinski PE, et al: MicroRNA-132mediated loss of p120RasGAP activates the endothelium to facilitate pathological angiogenesis. Nat Med 2010, 16(8):909-914.

22. Huang PH, Chen YH, Chen YL, Wu TC, Chen JW, Lin SJ: Vascular endothelial function and circulating endothelial progenitor cells in patients with cardiac syndrome X. Heart 2007, 93(9):1064-1070.

23. Chen YH, Lin SJ, Lin FY, Wu TC, Tsao CR, Huang PH, Liu PL, Chen YL, Chen JW: High glucose impairs early and late endothelial progenitor cells by modifying nitric oxide-related but not oxidative stress-mediated mechanisms. Diabetes 2007, 56(6):1559-1568.

24. Wang HW, Trotter MW, Lagos D, Bourboulia D, Henderson S, Makinen T, Elliman S, Flanagan AM, Alitalo K, Boshoff C: Kaposi sarcoma herpesvirusinduced cellular reprogramming contributes to the lymphatic endothelial gene expression in Kaposi sarcoma. Nat Genet 2004 36(7):687-693

25. Smyth GK: Linear models and empirical bayes methods for assessing differential expression in microarray experiments. Stat Appl Genet Mol Biol 2004, 3(1):Article3.

26. Storey JD, Tibshirani R: Statistical significance for genomewide studies. Proc Natl Acad Sci U S A 2003, 100(16):9440-9445. Epub 2003 Jul 9425

27. Yang TP, Chang TY, Lin CH, Hsu MT, Wang HW: ArrayFusion: a web application for multi-dimensional analysis of $\mathrm{CGH}$, SNP and microarray data. Bioinformatics 2006, 22(21):2697-2698.

28. Mortazavi A, Williams BA, McCue K, Schaeffer L, Wold B: Mapping and quantifying mammalian transcriptomes by RNA-Seq. Nat Methods 2008, 5 (7):621-628

29. Hsu JB, Chiu CM, Hsu SD, Huang WY, Chien CH, Lee TY, Huang HD: miRTar: an integrated system for identifying miRNA-target interactions in human. BMC Bioinformatics 2011, 12:300.

30. Pepini T, Gorbunova EE, Gavrilovskaya IN, Mackow JE, Mackow ER: Andes virus regulation of cellular microRNAs contributes to hantavirus-induced endothelial cell permeability. J Virol 2010, 84(22):11929-11936.

31. Zampetaki A, Kiechl S, Drozdov I, Willeit P, Mayr U, Prokopi M, Mayr A,

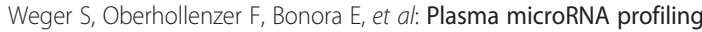
reveals loss of endothelial miR-126 and other microRNAs in type 2 diabetes. Circ Res 2010, 107(6):810-817.

32. Caporali A, Meloni M, Vollenkle C, Bonci D, Sala-Newby GB, Addis R, Spinetti G, Losa S, Masson R, Baker AH, et al: Deregulation of microRNA-503 contributes to diabetes mellitus-induced impairment of endothelial function and reparative angiogenesis after limb ischemia. Circulation 2011, 123(3):282-291

33. Huang TS, Hsieh JY, Wu YH, Jen CH, Tsuang YH, Chiou SH, Partanen J, Anderson $\mathrm{H}$, Jaatinen $\mathrm{T}, \mathrm{Yu}$ YH, et al: Functional network reconstruction reveals somatic stemness genetic maps and dedifferentiation-like transcriptome reprogramming induced by GATA2. Stem Cells 2008, 26(5):1186-1201.

34. Fiedler J, Jazbutyte V, Kirchmaier BC, Gupta SK, Lorenzen J, Hartmann D, Galuppo P, Kneitz S, Pena JT, Sohn-Lee C, et al: MicroRNA-24 regulates vascularity after myocardial infarction. Circulation 2011, 124(6):720-730.

35. Grundmann S, Hans FP, Kinniry S, Heinke J, Helbing T, Bluhm F, Sluijter JP, Hoefer I, Pasterkamp G, Bode C, et al: MicroRNA-100 regulates neovascularization by suppression of mammalian target of rapamycin in endothelial and vascular smooth muscle cells. Circulation 2011, 123(9):999-1009.

36. Fang JH, Zhou HC, Zeng C, Yang J, Liu Y, Huang X, Zhang JP, Guan XY, Zhuang SM: MicroRNA-29b suppresses tumor angiogenesis, invasion and metastasis by regulating MMP-2 expression. Hepatology 2011, 54(5):1729-1740.

37. Tsai YH, Wu MF, Wu YH, Chang SJ, Lin SF, Sharp TV, Wang HW: The M type $\mathrm{K} 15$ protein of Kaposi's sarcoma-associated herpesvirus regulates microRNA expression via its $\mathrm{SH} 2$-binding motif to induce cell migration and invasion. J Virol 2009, 83(2):622-632.

38. Real C, Caiado F, Dias S: Endothelial progenitors in vascular repair and angiogenesis: how many are needed and what to do? Cardiovasc Hematol Disord Drug Targets 2008, 8(3):185-193.

39. Fuchs S, Dohle E, Kolbe M, Kirkpatrick CJ: Outgrowth endothelial cells: sources, characteristics and potential applications in tissue engineering and regenerative medicine. Adv Biochem Eng Biotechnol 2010, 123:201-217.

40. Rafii S, Lyden D: Therapeutic stem and progenitor cell transplantation for organ vascularization and regeneration. Nat Med 2003, 9(6):702-712.

41. Wohrle J, Birkemeyer R, Markovic S, Nguyen TV, Sinha A, Miljak T, Spiess J, Rottbauer W, Rittger $\mathrm{H}$ : Prospective randomised trial evaluating a paclitaxel-coated balloon in patients treated with endothelial progenitor cell capturing stents for de novo coronary artery disease. Heart 2011, 97(16):1338-1342.

42. Zorn AM, Butler K, Gurdon JB: Anterior endomesoderm specification in Xenopus by $\mathrm{Wnt} /$ beta-catenin and TGF-beta signalling pathways. Dev Biol 1999, 209(2):282-297.

43. Gherghe CM, Duan J, Gong J, Rojas M, Klauber-Demore N, Majesky M, Deb A: Wnt1 is a proangiogenic molecule, enhances human endothelial progenitor function, and increases blood flow to ischemic limbs in a HGF-dependent manner. Faseb J 2011, 25(6):1836-1843.

44. Wang Z, Gerstein M, Snyder M: RNA-Seq: a revolutionary tool for transcriptomics. Nat Rev Genet 2009, 10(1):57-63.

45. Pedrioli DM, Karpanen T, Dabouras $V$, Jurisic G, van de Hoek G, Shin JW, Marino D, Kalin RE, Leidel S, Cinelli $P$, et al: miR-31 functions as a negative regulator of lymphatic vascular lineage-specific differentiation in vitro and vascular development in vivo. Mol Cell Biol 2010, 30(14):3620-3634

46. Menghini $R$, Casagrande V, Cardellini M, Martelli E, Terrinoni A, Amati F, Vasa-Nicotera M, Ippoliti A, Novelli G, Melino G, et al: MicroRNA 217 modulates endothelial cell senescence via silent information regulator 1 . Circulation 2009, 120(15):1524-1532.

47. Wang XH, Qian RZ, Zhang W, Chen SF, Jin HM, Hu RM: MicroRNA-320 expression in myocardial microvascular endothelial cells and its relationship with insulin-like growth factor- 1 in type 2 diabetic rats. Clin Exp Pharmacol Physiol 2009, 36(2):181-188.

48. Fang L, Deng Z, Shatseva T, Yang J, Peng C, Du WW, Yee AJ, Ang LC, He C, Shan SW, et al: MicroRNA miR-93 promotes tumor growth and angiogenesis by targeting integrin-beta8. Oncogene 2011, 30(7):806-821.

49. Li D, Yang P, Xiong Q, Song X, Yang X, Liu L, Yuan W, Rui YC: MicroRNA$125 \mathrm{a} / \mathrm{b}-5 \mathrm{p}$ inhibits endothelin-1 expression in vascular endothelial cells. J Hypertens 2010, 28(8):1646-1654.

50. Fang Y, Shi C, Manduchi E, Civelek M, Davies PF: MicroRNA-10a regulation of proinflammatory phenotype in athero-susceptible endothelium in vivo and in vitro. Proc Natl Acad Sci U S A 2010, 107(30):13450-13455.

doi:10.1186/1471-2164-13-447

Cite this article as: Cheng et al:: Genetic module and miRNome trait analyses reflect the distinct biological features of endothelial progenitor cells from different anatomic locations. BMC Genomics 2012 13:447. 